\title{
FGF21 facilitates autophagy in prostate cancer cells by inhibiting the Pl3K-Akt-mTOR signaling pathway
}

Han Dai ${ }^{1,2}$, Wenjing Hư ${ }^{3}$, Lianying Zhang ${ }^{1}$, Feiyu Jiang ${ }^{1}$, Xiongmin Mao ${ }^{1}$, Gangyi Yang ${ }^{3}$ and Ling Li (1)

\begin{abstract}
Fibroblast growth factor 21 (FGF21) plays an important role in regulating glucose and lipid metabolism, but its role in cancer is less well-studied. We aimed to investigate the action of FGF21 in the development of prostate cancer (PCa). Herein, we found that FGF21 expression was markedly downregulated in PCa tissues and cell lines. FGF21 inhibited the proliferation and clone formation of LNCaP cells (a PCa cell line) and promoted apoptosis. FGF21 also inhibited PCa cell migration and invasiveness. The Gene Ontology and Kyoto Encyclopedia of Genes and Genomes analyses revealed that FGF21 was related to autophagy and the phosphatidylinositol 3-kinase-Akt kinase-mammalian target of rapamycin (PI3K-Akt-mTOR) pathway. Mechanistically, FGF21 promoted autophagy in LNCaP cells by inhibiting the PI3K-Akt-mTOR-70S6K pathway. In addition, FGF21 inhibited PCa tumorigenesis in vivo in nude mice. Altogether, our findings show that FGF21 inhibits PCa cell proliferation and promoted apoptosis in PCa cells through facilitated autophagy. Therefore, FGF21 might be a potential novel target in PCa therapy.
\end{abstract}

\section{Introduction}

Prostate cancer ( $\mathrm{PCa}$ ) is one of the most common malignant tumors and is an important cause of cancerrelated deaths in men. It also has the highest incidence rate among all types of malignant tumors in older men ${ }^{1}$. In recent decades, the incidence and mortality rate of $\mathrm{PCa}$ has increased in China ${ }^{2}$. Castration treatment can improve the prognosis of PCa; however, many patients will nevertheless progress to castration-resistant $\mathrm{PCa}^{3}$. Therefore, there is an urgent need for more effective $\mathrm{PCa}$ treatments.

The fibroblast growth factor (FGF) family is composed of 22 members, which can be divided into typical FGFs

\footnotetext{
Correspondence: Ling Li (liling@cqmu.edu.cn)

${ }^{1}$ The Key Laboratory of Laboratory Medical Diagnostics in the Ministry of Education and Department of Clinical Biochemistry, College of Laboratory Medicine, Chongqing Medical University, Chongqing, China

${ }^{2}$ Department of Endocrinology, The Second Affiliated Hospital, Chongqing Medical University, Chongaing, China

Full list of author information is available at the end of the article

These authors contributed equally: Han Dai, Wenjing Hu

Edited by B. Zhivotovsky
}

and hormone-like FGFs ${ }^{4}$. FGFs play an important role in cell growth and differentiation, angiogenesis, embryonic development, wound healing and repair, and metabolic regulation ${ }^{5}$. FGF21 is a member of the FGF ligand family and is mainly secreted by the liver. Like the gut-derived FGF15 and the bone-derived FGF23, FGF21 is involved in maintaining metabolic homeostasis ${ }^{6}$ (e.g., bile acid metabolism ${ }^{7}$, glucose and lipid metabolism, among others) and is related to the metabolic adaptation of the fasting state ${ }^{8-10}$. Under physiological conditions, the liver secretes a large amount of FGF21 protein into the circulation. In addition, FGF21 is also expressed in other tissues such as the pancreas, adipose tissue, muscle, and kidney under pathological and stress conditions ${ }^{11}$. Recent studies have also revealed that FGF21 is associated with the occurrence and development of tumors such as colon cancer $^{12}$, thyroid cancer ${ }^{11}$, and liver cancer ${ }^{13}$. However, its role in PCa has not been reported to date. Moreover, although previous studies have shown that FGF19 and FGF23 can promote the occurrence and progression of $\mathrm{PCa}^{14,15}$, their underlying mechanisms are unclear. As

\section{(c) The Author(s) 2021}

(c) (i) Open Access This article is licensed under a Creative Commons Attribution 4.0 International License, which permits use, sharing, adaptation, distribution and reproduction cc) in any medium or format, as long as you give appropriate credit to the original author(s) and the source, provide a link to the Creative Commons license, and indicate if changes were made. The images or other third party material in this article are included in the article's Creative Commons license, unless indicated otherwise in a credit line to the material. If material is not included in the article's Creative Commons license and your intended use is not permitted by statutory regulation or exceeds the permitted use, you will need to obtain permission directly from the copyright holder. To view a copy of this license, visit http://creativecommons.org/licenses/by/4.0/. 
FGF21 has a different molecular structure and tissue source from FGF19 and FGF23 and plays an essential role in metabolism in vivo, we speculate that FGF21 plays a distinct role in $\mathrm{PCa}$ occurrence and development. Here, we investigated the effects of FGF21 on PCa cell proliferation, migration, invasion, apoptosis, and autophagy, and its possible mechanisms.

\section{Materials and methods}

\section{Tissue samples from patients}

We obtained tissue specimens from 42 patients with $\mathrm{PCa}$ and 24 patients with benign prostatic hyperplasia $(\mathrm{BPH})$ who had undergone surgery at the Second Affiliated Hospital, Chongqing Medical University (Chongqing, China), from March 2018 to March 2019. Two pathologists examined paraffin sections according to the World Health Organization (WHO) diagnostic criteria for the histological classification of $\mathrm{PCa}^{16}$. The $\mathrm{PCa}$ specimens were classified according to 2016 WHO Gleason score for $\mathrm{PCa}$, one of the strongest clinical predictors of PCa progression and outcomes. The Gleason score stratifies $\mathrm{PCa}$ based on prostate-specific antigen, clinical stage, and biopsy score into low, intermediate, or high risk, where both $3+4=7$ and $4+3=7$ are considered the same within the intermediate-risk group. The specific groupings of the PCa tissues were as follows: 18 patients had Gleason score $\leq 7$ and 24 patients had Gleason score $>7^{17}$. The Chongqing Medical University Ethics Committee approved this study (2017-34), and informed consent was obtained from all participants.

\section{Gene Ontology (GO) and Kyoto Encyclopedia of Genes and Genomes (KEGG) enrichment analysis}

Gene expression profiles from GSE33684 were downloaded from the Gene Expression Omnibus (GEO) online database (https://www.ncbi.nlm.nih.gov/geo/). GSE33684 is a microarray dataset from diet-induced obesity wildtype (WT) and FGF21 knockout (KO) mice. Differential expression genes (DEGs) were analyzed with the Limma software package (R3.6.1). Genes with $|\log 2 \mathrm{FC}|>1$ and adjusted $p$ value $<0.05$ were identified as DEGs (i.e., their difference multiple was $>2$ ), indicating that their expression is closely related to FGF21. The lists of the GO and KEGG analyses were obtained through enrichment analysis. The molecular functions, biological processes, and cellular components of the genes were obtained. A $p$ value of $<0.05$ was considered statistically significant ${ }^{18}$.

\section{Recombinant plasmid construction, cell culture, and treatments}

LNCaP, 22Rv1, DU145, PC3, and RWPE-1 cells (American Type Culture Collection, Manassas, VA, USA) were maintained in RPMI-1640 medium. The PC3 cells were cultured in Dulbecco's modified Eagle's medium Nutrient
Mixture F-12. All medium was supplemented with $10 \%$ fetal bovine serum and $1 \%$ penicillin/streptomycin.

Recombinant plasmids expressing FGF21 (pEGFPFGF21, p-FGF21) and empty plasmids (pEGFP-N1, p-N1) were constructed and verified by $X h o I$ and EcoRI digestion and DNA sequencing, as previously reported ${ }^{19}$. To investigate the effect of FGF21 on PCa cells, we transfected LNCaP cells with p-FGF21 or p-N1 for $72 \mathrm{~h}$, and then incubated them with 5 or $25 \mathrm{mM}$ glucose for $48 \mathrm{~h}$. For mammalian target of rapamycin (mTOR) signaling pathway analysis, LNCaP cells that had been transfected with $\mathrm{p}-F G F 21$ or $\mathrm{p}-\mathrm{N} 1$ were treated with or without MHY1485, an mTOR agonist, for $24 \mathrm{~h}$.

\section{Cell viability and focus formation assay}

Cell viability was examined using Cell Counting Kit-8 (CCK-8) according to the manufacturer's instructions, as previously reported ${ }^{20}$. The viability of the control group was deemed $100 \%$ survival. For the focus formation experiment, LNCaP cells were transfected with p-FGF21 or p-N1, and seeded into a 6-well plate. The cells were then cultured with glucose $\left(5\right.$ or $25 \mathrm{mM}$ ) at $37^{\circ} \mathrm{C}$ for 10 days. Crystal violet staining was used for counting the cell colonies. The experiments were repeated at least three times.

\section{Wound-healing and transwell migration experiments}

LNCaP cells that had been transfected with p-FGF21 or $\mathrm{p}-\mathrm{N} 1$ were seeded into 6 -well culture plates. For the wound-healing experiment, a straight scratch was made in the cell monolayer with a pipette tip when the cells were 90\% confluent. The cells were cultured in RPMI-1640 medium with 5 or $25 \mathrm{mM}$ glucose for $24 \mathrm{~h}$. The scratch was examined and photographed under a light microscope, and the cell-free area was quantified using the ImageJ software.

For the Transwell migration assay, the cells were seeded into the upper chamber of 24-well Transwell plates with $200 \mu \mathrm{L}$ medium. Then, the lower chamber was filled with $600 \mu \mathrm{L} \mathrm{10 \%} \mathrm{fetal} \mathrm{bovine} \mathrm{serum-supplemented} \mathrm{medium,}$ and the cells were cultured for $12 \mathrm{~h}$. Subsequently, the cells on the upper surface of the chamber were removed using a cotton swab. The cells on the bottom surface were fixed in $4 \%$ paraformaldehyde (PFA) for $30 \mathrm{~min}$ and stained with $0.1 \%$ crystal violet for $10 \mathrm{~min}$. The migrated cells were photographed under a microscope, and the images were analyzed using ImageJ.

\section{Flow cytometry analysis}

LNCaP cells were seeded and cultured in 6-well plates for $48 \mathrm{~h}$ at $37^{\circ} \mathrm{C}$, and then transfected with p-FGF21 or $\mathrm{p}-\mathrm{N} 1$ for $72 \mathrm{~h}$. The cells were collected and washed in icecold phosphate-buffered saline, then stained with annexin V-allophycocyanin (APC-A) and 4', 6-diamidino-2phenylindole (DAPI) PB450-A from an Apoptosis 
Detection Kit (BD Biosciences Inc., San Jose, CA, USA) according to the manufacturer's instructions. For determining the cell cycle phase, the cells were fixed in $75 \%$ icecold ethanol overnight and then treated with $1 \mathrm{mg} / \mathrm{mL}$ RNase (Sigma-Aldrich) for $10 \mathrm{~min}$ at $37^{\circ} \mathrm{C}$. DNA was stained with propidium iodide $(15 \mathrm{mg} / \mathrm{mL})$ for $20 \mathrm{~min}$ at $4{ }^{\circ} \mathrm{C}$ in the dark. Cell cycle profiles and apoptosis were analyzed by flow cytometry (FACS Vantage SE, BD, Franklin Lakes, NJ, USA).

\section{Tumorigenicity experiments in nude mice}

For the tumorigenicity experiments, we used 5-week-old male BALB/c nude mice from Huafukang Biotechnology Co., Ltd (Beijing, China). LNCaP cells infected with p-FGF21 or p-N1 $\left(5 \times 10^{6}\right.$ cells, $\left.200 \mu \mathrm{L}\right)$ were injected subcutaneously into the dorsal thighs of the mice. Tumor growth was observed for 3 weeks, and the tumor volume was calculated as $V=$ length $\times$ width $^{2} \times 0.5$. After 3 weeks, the mice were sacrificed, and the tumor tissue was collected and frozen in liquid nitrogen for subsequent analysis. All animal experiments were supported by the Chongqing Medical University Animal Experimentation Ethics Committee (2018-048).

\section{Hematoxylin-eosin (H\&E) and immunohistochemistry staining}

H\&E staining was performed according to routine protocols $^{21}$. For immunohistochemistry (IHC) staining, tumor tissues from the patients with $\mathrm{PCa}$ and $\mathrm{BPH}$ were fixed in $4 \%$ PFA at $4{ }^{\circ} \mathrm{C}$ overnight, embedded in paraffin, and sectioned to $7 \mu \mathrm{m}$ thickness. IHC was performed using an antibody against FGF21 (1:250, ab171941, Abcam, Cambridge, UK).

\section{Immunofluorescence assays}

LNCaP cells were transfected with $\mathrm{p}-F G F 21$ or $\mathrm{p}-\mathrm{N} 1$ and cultured for $72 \mathrm{~h}$. The cells were incubated with $0.1 \%$ Triton X-100 at room temperature (RT) for $15 \mathrm{~min}$, and then blocked with $5 \%$ goat serum at $37{ }^{\circ} \mathrm{C}$ for $30 \mathrm{~min}$. Next, the cells were incubated with primary antibody against LC3B (1:200, NB100-2220, Novus Biologicals, Colorado, USA) at $4{ }^{\circ} \mathrm{C}$ overnight, and then with the secondary antibody at RT for $1 \mathrm{~h}$, before being stained with DAPI for $1 \mathrm{~min}$.

\section{Transmission electron microscopy (TEM)}

LNCaP cells were fixed in $2.5 \%$ glutaraldehyde for $1 \mathrm{~h}$ and washed twice with $0.1 \mathrm{~mol} / \mathrm{L}$ sodium cacodylate. The cells were then fixed in $2 \%$ osmium tetroxide for $2 \mathrm{~h}$, washed with distilled water, and stained with $0.5 \%$ uranyl acetate. Next, the cells were dehydrated in ethanol, rinsed in propylene oxide, and embedded in 1:1 propylene oxide: Spurr's resin for $24 \mathrm{~h}$. Ultrathin sections $(80 \mathrm{~nm})$ were cut with an ultra-microtome. Images were obtained under a Hitachi HT-7700 TEM (Hitachi, Tokyo, Japan).

\section{Quantitative RT-PCR (qRT-PCR) and western blotting}

qRT-PCR was performed as previously described ${ }^{22}$. The primer pairs are listed in Table S1. Western blot analysis was performed as described previously ${ }^{23}$. The primary antibodies included anti-LC3 (1:1000, NB100-2220, Novus Biologicals, Colorado, USA), anti-FGF21 (1:1000, ab171941, Abcam, Cambridge, UK), anti-P62 (1:1000, ab56416, Abcam, Cambridge, UK), anti-Ki-67 (1:1000, ab16667, Abcam, Cambridge, UK), anti-LAMP2 (1:500, ab13524, Abcam, Cambridge, UK), anti-BCL-2 (1:1000, \#15071T, Cell Signaling Technology, Massachusetts, USA), anti-Akt kinase (Akt; 1:1000, \#9272s, Cell Signaling Technology, Massachusetts, USA), phospho-Akt (Ser 473; 1:1000, \#4060s, Cell Signaling Technology, Massachusetts, USA), anti-mTOR (1:1000, \#2972s, Cell Signaling Technology, Massachusetts, USA), phospho-mTOR (1:1000, \#2971s, Cell Signaling Technology, Massachusetts, USA), anti-p70S6K (1:1000, \#2708s, Cell Signaling Technology, Massachusetts, USA), phospho-p70S6K (Thr389; 1:1000, \#9205s, Cell Signaling Technology, Massachusetts, USA), and anti- $\beta$-actin (1:1000, TA-09, ZSGB-BIO, Beijing, China).

\section{Statistical analysis}

Data were expressed as the mean \pm SD or SE. GraphPad Prism software (version 6.0) was used to draw bar and line charts. The differences between the groups were assessed by a two-way ANOVA with a post hoc test. The two groups were compared by the Student's $t$ test. Statistical analyses were performed using SPSS software (version 19.0, Chicago, IL, USA). A $p$ value $<0.05$ was considered statistically significant.

\section{Results}

Expression of FGF21 in PCa tissues and cell lines

We performed H\&E and FGF21 IHC staining of tissues from 42 patients with $\mathrm{PCa}$ and 24 patients with $\mathrm{BPH}$. The patients with PCa had lower FGF21 expression levels than the patients with $\mathrm{BPH}$ (Fig. 1A).

Further, we measured FGF21 mRNA and protein expression in prostate epithelial cells (RWPE-1) and PCa cell lines (i.e., LNCaP, PC3, DU145, 22Rv1) using RT-PCR and western blotting. All PCa cell lines had significantly lower FGF21 mRNA expression than the RWPE-1 cells (Fig. 1B). Moreover, all PCa cell lines had significantly lower FGF21 protein expression than the RWPE-1 cells (Fig. 1C, D). As LNCaP cells showed the lowest FGF21 protein expression levels, we selected that cell line for further study.

\section{Association of FGF21 with the pathological features of patients with $\mathrm{PCa}$}

We analyzed the relationship between FGF21 expression and the clinical characteristics of the patients with 
A

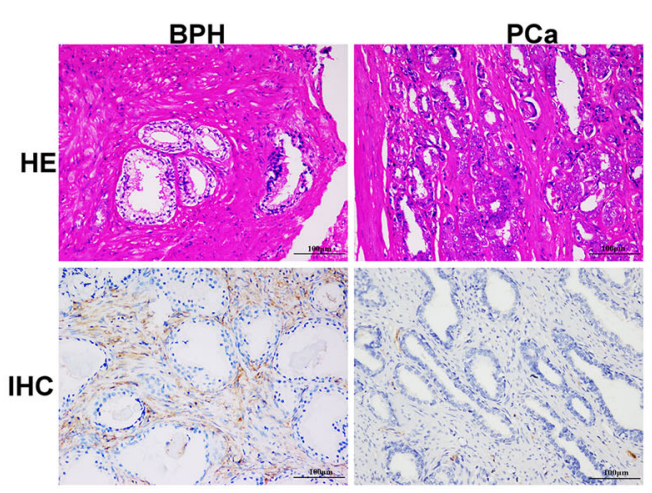

C

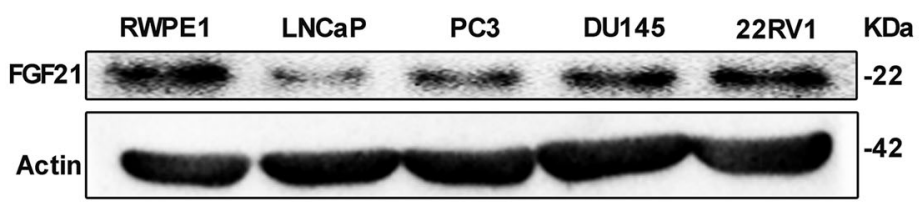

B

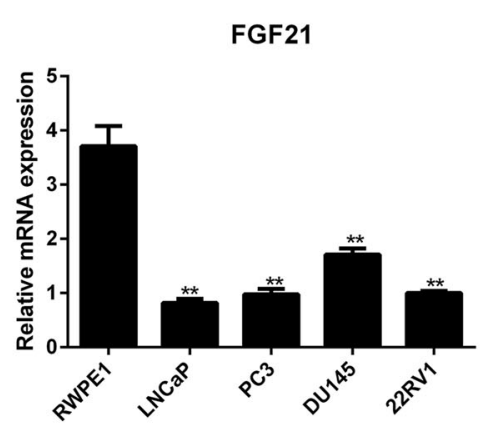

D

FGF21

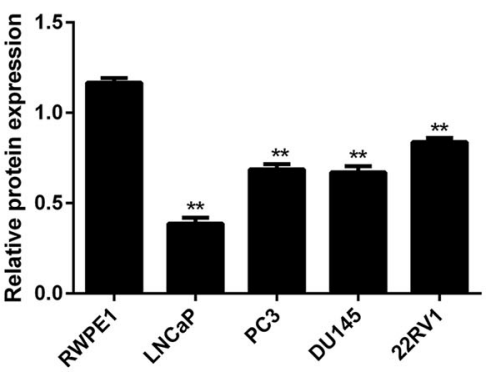

Fig. 1 Expression of FGF21 in prostate cancer tissues and cell lines. A H\&E and FGF21 IHC staining in tissues from PCa and BPH. B, C FGF21 mRNA (B) and protein (C) expression in different PCa cell lines. D Quantitative analysis of FGF21 protein expression. RWPE-1, prostate epithelial cells. Data were expressed as the mean \pm SD. ${ }^{*} p<0.01$ vs. RWPE-1.

PCa. Table S2 shows that FGF21 expression was closely related to the pathological stage and Gleason score, suggesting that it might be involved in the development of PCa.

\section{FGF21 inhibits LNCaP cell proliferation and clone formation and promotes apoptosis}

To investigate the effects of FGF21 on cell proliferation and clone formation in vitro, we transfected LNCaP cells with p-FGF21 or p-N1. Further, as recent studies have shown that high glucose levels promote tumor cell proliferation and migration ${ }^{24}$, we cultured the cells in low $(5 \mathrm{mM})$ and high $(25 \mathrm{mM})$ levels of glucose. As expected, FGF21 expression was significantly increased at both mRNA and protein levels in the p-FGF21-transfected LNCaP cells (Fig. 2A, B).

Next, we used CCK-8 to detect the effect of FGF21 on LNCaP cell proliferation under different glucose concentrations. The proliferative ability of LNCaP cells increased significantly in hyperglycemic conditions (i.e., $25 \mathrm{mM}$ glucose) compared with low-glucose (5 mM glucose) culture. However, the proliferative ability of the pFGF21 cells was significantly decreased (Fig. 2C).

We also examined the impact of FGF21 on LNCaP cell apoptosis by flow cytometry. LNCaP cells in hyperglycemic conditions had decreased levels of apoptosis than the cells in hypoglycemic conditions. FGF21 overexpression significantly increased LNCaP cell apoptosis under both hypoglycemic and hyperglycemic conditions (Fig. 2D). Furthermore, FGF21 overexpression significantly inhibited the hyperglycemia-induced clonal formation in the LNCaP cells (Fig. 2E). qRT-PCR and western blotting showed that FGF21 overexpression significantly inhibited the mRNA and protein expression of the cell proliferation and anti-apoptosis markers, including Ki-67 and BCL-2 (Fig. 2F, G). These data indicate that FGF21 inhibits LNCaP cell proliferation and clone formation, and promotes apoptosis, especially under highglucose conditions.

\section{FGF21 inhibits LNCaP cell migration and invasiveness}

We observed the impact of FGF21 on LNCaP cell migration and invasiveness with wound-healing and Transwell migration assays. The wound-healing assay showed LNCaP cell mobility was increased under hyperglycemic conditions (Fig. 3A). However, compared with $\mathrm{p}-N 1$ treatment, $\mathrm{p}-F G F 21$ treatment led to a significant reduction in the mobility of LNCaP cells cultured with $25 \mathrm{mM}$ glucose (Fig. 3A). In the Transwell migration experiments, LNCaP cells exposed to 


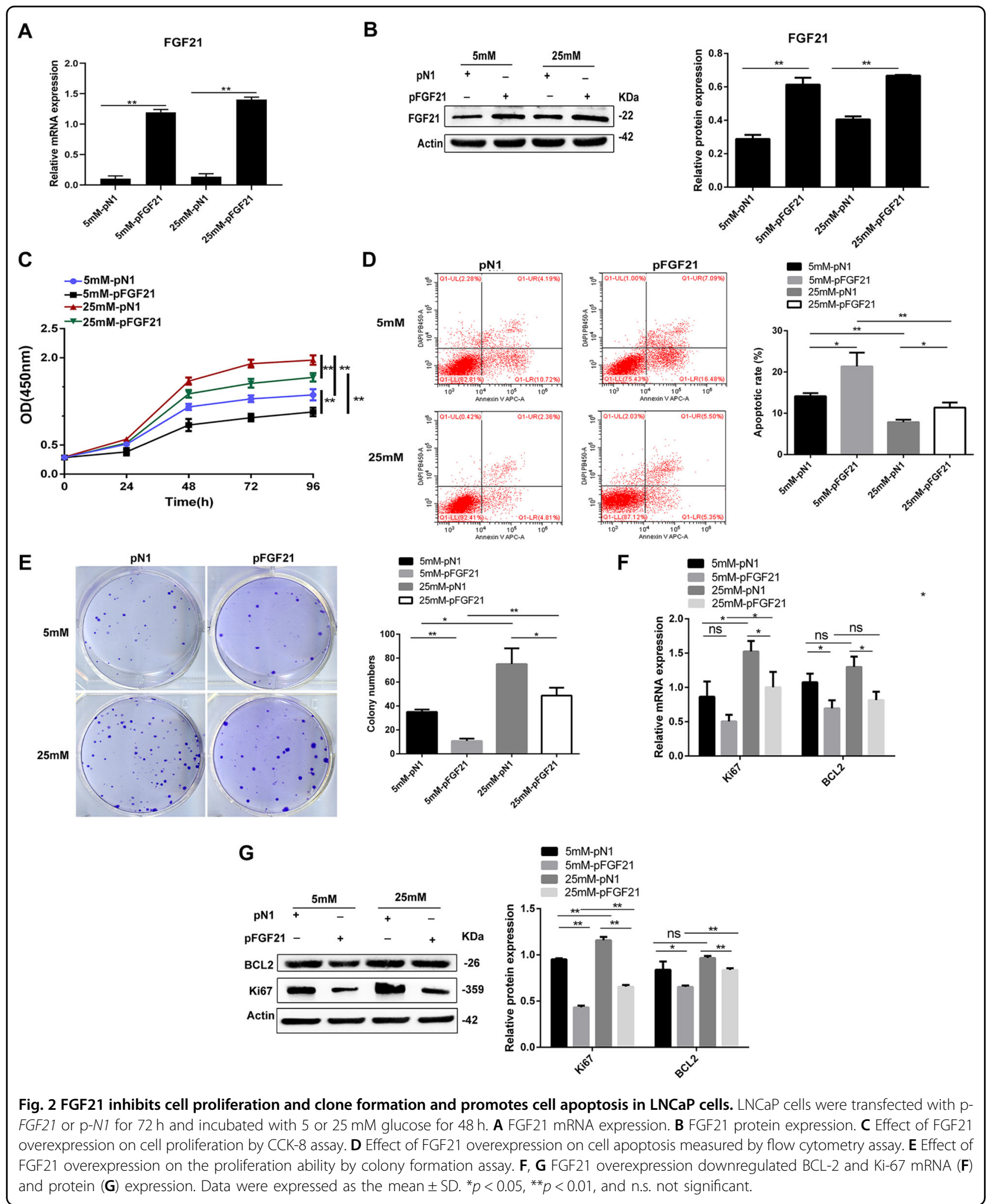

hyperglycemic conditions had significantly increased numbers of invaded cells. However, p-FGF21 transfection reduced the number of invaded cells under both high- and low-glucose conditions (Fig. 3B). These results suggest that $\mathrm{p}-F G F 21$ inhibits $\mathrm{LNCaP}$ cell migration and invasiveness. 


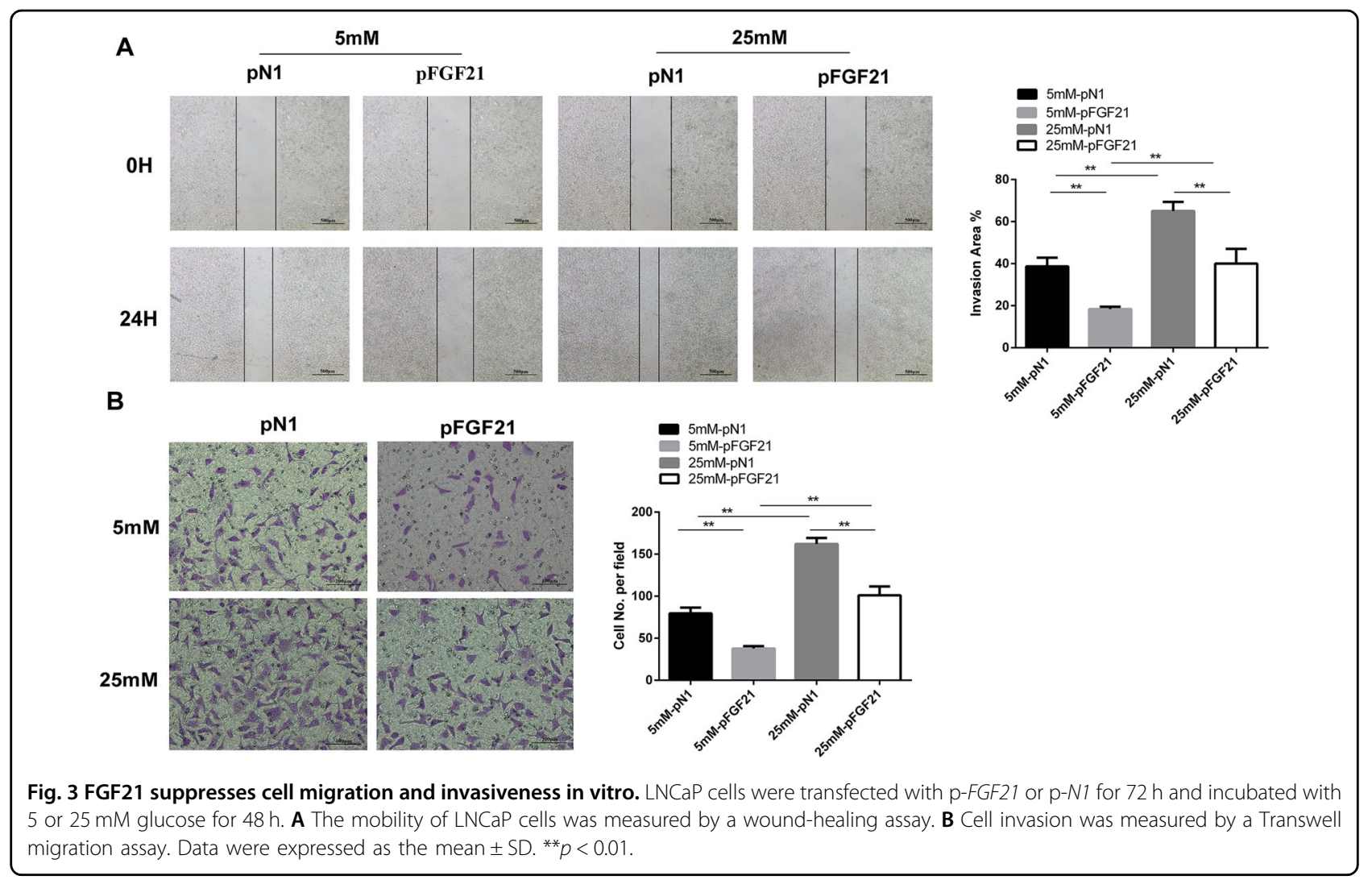

\section{Identification of the genes and signaling pathways related to FGF21-mediated apoptosis}

To determine the underlying mechanism of FGF21mediated apoptosis, we analyzed gene expression profiles and candidate signal pathways from a GEO dataset (GSE33684). GO analysis showed that among 13 DEGs, nine were upregulated, and four were downregulated (Fig. 4A). These genes are involved in the cellular response to interferon-beta 2 (IFN- $\beta 2$ ), response to IFN, and positive regulation of cold-induced thermogenesis, among others (Fig. 4B and Table S3). Of these 13 DEGs, thrombospondin2 (THBS2) was related to B cell lymphoma 2 (BCL-2) (an apoptotic protein), and autophagy ${ }^{25}$.

KEGG pathway analysis showed that FGF21 is mainly related to the following signaling pathways: the phosphatidylinositol 3-kinase (PI3K)/Akt/mTOR, folate biosynthesis, and fructose and mannose metabolism (Fig. $4 \mathrm{C}$ and Table S4). Previous studies have found that the PI3K-Akt-mTOR signaling pathway is associated with autophagy ${ }^{26}$. Therefore, these results suggest that FGF21 is related to the PI3K-Akt-mTOR signaling pathway and autophagy.

\section{FGF21 promotes autophagy in LNCaP cells}

Previous studies have shown that the activation of autophagy inhibits cell proliferation ${ }^{27}$, and our GO and KEGG pathway analyses showed that FGF21 is related to autophagy.
Therefore, we studied the effect of FGF21 on autophagy in vitro to investigate the mechanisms underlying the FGF21mediated inhibition of cell proliferation. First, we examined the expression of LC3B (a central protein in the autophagy pathway) in LNCaP cells via immunofluorescence staining. Figure $5 \mathrm{~A}$ shows that there were reduced levels of LC3B in LNCaP cells under hyperglycemic conditions relative to hypoglycemic conditions. However, in the p-FGF21 cells, immunofluorescence staining of LC3B was increased (Fig. 5A). Second, TEM showed that there were fewer autophagosomes in LNCaP cells under hyperglycemic conditions than that in hypoglycemic conditions. The number of autophagosomes in the p-FGF21 cells was increased under both low- and high-glucose conditions (Fig. 5B).

To confirm the effect of FGF21 on autophagy, we used qRT-PCR and western blotting to examine the expression of LC3-I/II, p62, and LAMP2, three autophagy markers, in LNCaP cells. The overexpression of FGF21 increased LC3-II and LAMP2 expression and decreased p62 expression at mRNA and protein levels in the LNCaP cells (Fig. 5C, D). These data indicate that FGF21 increases the conversion of LC3-I to LC3-II and promotes autophagy in these cells.

FGF21 inhibits PI3K-Akt-mTOR signaling in LNCaP cells

KEGG pathway analysis showed that FGF21 is related to PI3K-Akt-mTOR signaling. Therefore, we investigated 

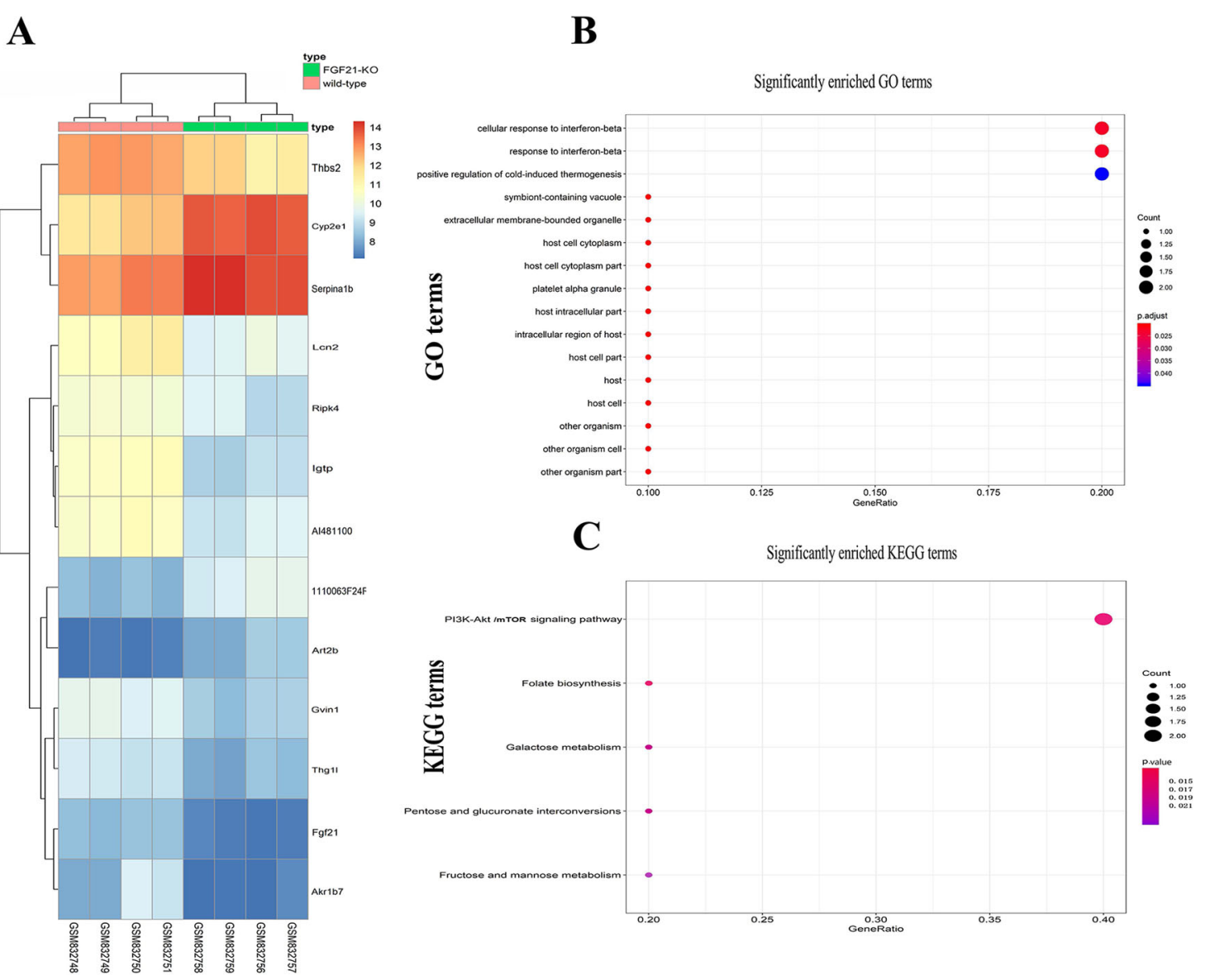

Fig. 4 Identification of genes and signal pathways related to FGF21. A The heat maps for DEGs. Red and blue colors indicate relative expression above or below the average. B Functional analysis of DEGs. C KEGG pathway analysis for 13 DEGs. More red color indicates the smaller $p$ values. DEG differentially expressed gene.

the effects of FGF21 on the PI3K-Akt-mTOR pathway in LNCaP cells to determine how FGF21 can regulate autophagy in PCa. Under hyperglycemic conditions, the phosphorylation of Akt, mTOR, and p70S6K (a downstream target activated by mTOR complex 1 [mTORC1]) was increased in LNCaP cells (Fig. 5E). However, in the FGF21-overexpressing LNCaP cells, the effects of high glucose on Akt, mTOR, and p70S6K phosphorylation levels were reduced (Fig. 5E).

To confirm that FGF21-mediated autophagy is driven by mTOR signaling, we transfected LNCaP cells with $\mathrm{p}$ N1 or p-FGF21 in the presence or absence of MHY1485, an mTORC1 agonist. mTOR activation blocked the FGF21-induced reduction of mTOR and p70S6K phosphorylation, and the downregulation of BCL-2, Ki-67, and p62 in the cells (Fig. 5F). In addition, MHY1485 inhibited the increased LAMP2 expression induced by FGF21 and attenuated the FGF21-induced conversion of LC3-I to LC3-II (Fig. 5F). These data indicate that FGF21 inhibits PI3K-Akt-mTOR signaling and facilitates autophagy.

\section{FGF21 inhibits PCa tumorigenesis in vivo}

We studied the impact of FGF21 on tumorigenesis in vivo using $\mathrm{PCa}$ xenograft models. Nude mice were subcutaneously implanted with $\mathrm{p}-\mathrm{N1}$ - or p-FGF21transfected LNCaP cells (at concentrations of 5 and $25 \mathrm{mM}$ D-glucose). The mice injected with high glucose-treated LNCaP cells had greater tumor volumes than the mice injected with the low glucosetreated LNCaP cells (Figure 6A-C). In addition, under both high- or low-glucose conditions 19 days after transplantation, the mice injected with $\mathrm{p}-F G F 21$ cells had smaller tumor volumes than the mice implanted with p-N1 cells (Fig. 6A, B). Furthermore, the tumors from the p-FGF21 cells were markedly smaller and lighter than those from p-N1 cells (Fig. 6C, D). When the transplanted cells were cultured with $25 \mathrm{mM}$ D-glucose, the inhibitory effect of p-FGF21 on the tumor was more obvious (Fig. 6C, D). These data indicate that FGF21 overexpression inhibits the growth of $\mathrm{PCa}$ in vivo. 


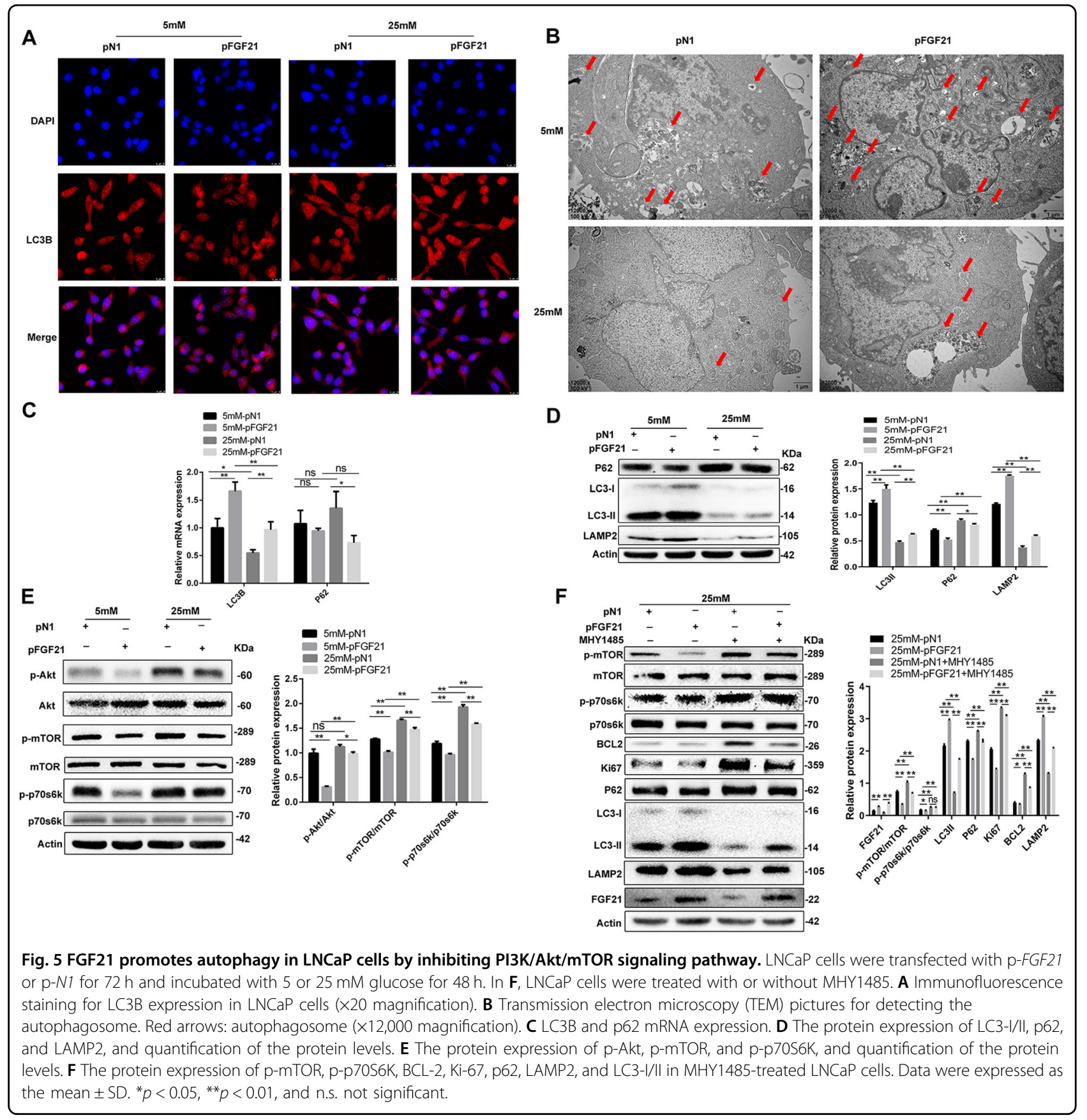

\section{Discussion}

Although previous studies have shown that FGFs derived from tumor cells or stromal cells induce tumor progression in several cancer types ${ }^{28-31}$, their precise role remains unclear. A recent study revealed that FGF21 mediates invasion and metabolic disorders in thyroid cancer and that elevated serum FGF21 levels in patients with thyroid cancer may be a marker of tumor progression $^{12}$. However, the role of FGF21 in the occurrence and development of PCa is unknown.
Here, we demonstrate that: (1) FGF21 expression is decreased in both PCa tissues and cell lines; (2) FGF21 inhibits the proliferation, clone formation, migration, and invasiveness of LNCaP cells (a PCa cell line) and promotes their apoptosis; (3) FGF21 overexpression attenuates high glucose-induced LNCaP cell proliferation and apoptosis; (4) FGF21 is related to autophagy and the PI3K-Akt-mTOR pathway; and (4) FGF21 increases autophagy by inhibiting the PI3K-Akt-mTOR signaling pathway. 


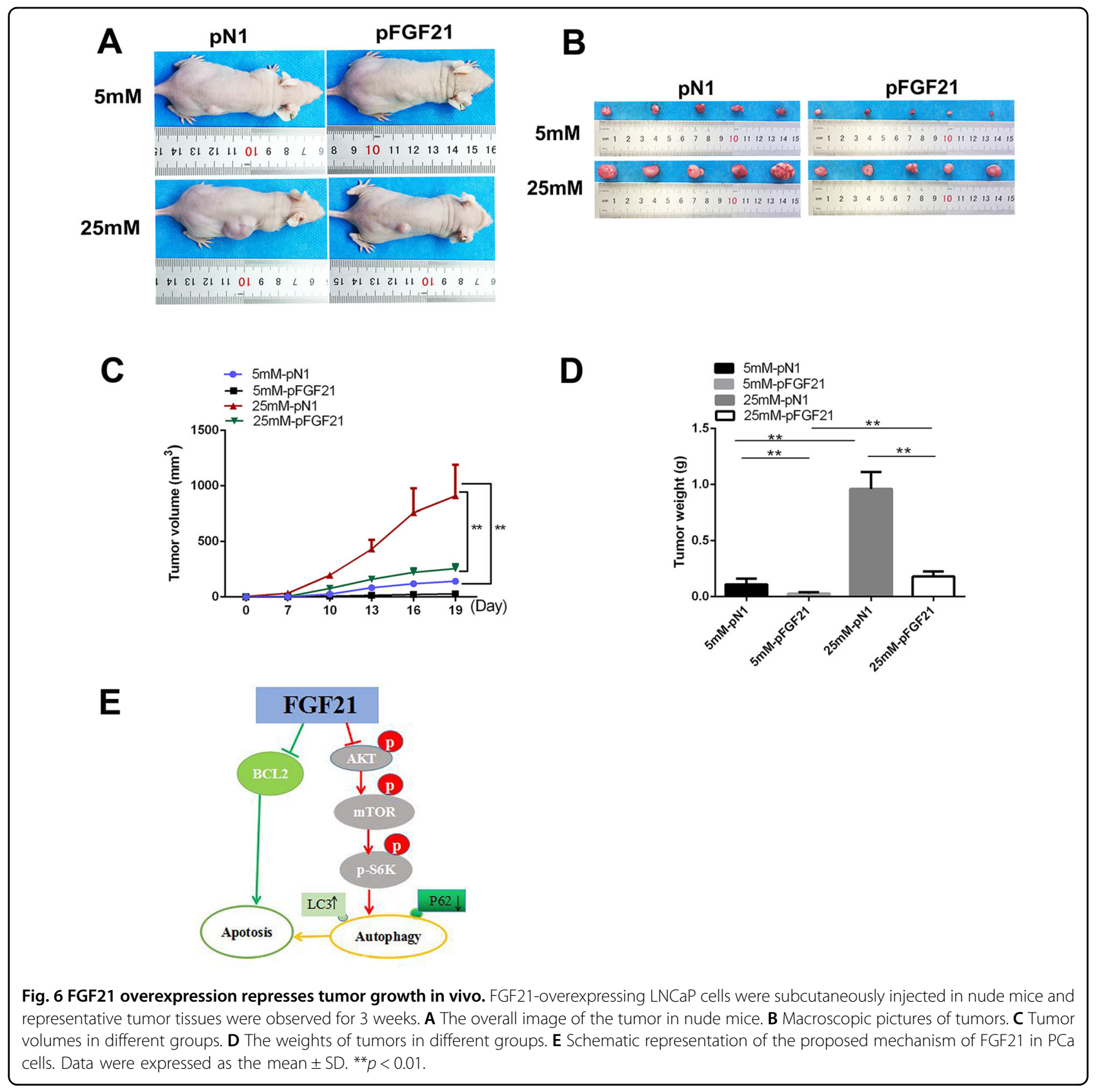

FGF21 is a cytokine derived from metabolism-related tissues (e.g., the liver, muscle, adipose tissue) and plays an important role in regulating glucose and lipid metabolism and metabolic adaptation in vivo. As tumor cells are metabolically active and vulnerable to nutrient starvation $^{32}$, FGF21 may affect their phenotype. Indeed, in the current study, we found that FGF21 inhibited LNCaP cell proliferation, clone formation, migration, and invasiveness, and promoted apoptosis. Our results are similar to two recent in vivo studies that found hepatic FGF21 expression was activated, and serum FGF21 levels were increased in response to hepatocarcinogenesis, breast cancer, primary renal tumors, and papillary thyroid cancer $^{12,33-35}$. These were mostly cross-sectional, population-based studies examining phenotypic characteristics, and without in-depth molecular biology studies. In those studies, the increased circulating levels of FGF21 observed in the patients with tumor might have been due to the increased synthesis and release of FGF21 in tumor cells, or the metabolic disorder of the microenvironment caused by tumor stress ${ }^{35}$. Therefore, in the present study, although we did not measure the circulating FGF21 levels, we believe that circulating FGF21 levels are increased in patients with PCa. 
The tumor microenvironment plays an important role in tumor cell growth. High glucose promotes tumor cell proliferation and migration ${ }^{25}$. Given the role of FGF21 in glucose metabolism, we investigated its effects on LNCaP cell proliferation and migration under high-glucose conditions. We found that FGF21 overexpression significantly inhibited the proliferation and migration of $\mathrm{LNCaP}$ cells induced by high glucose. Herein, the effect of FGF21 on glucose metabolism in LNCaP cells might be similar to that in previous reports of obese and type 2 diabetes mellitus populations and animals. Those studies reported that FGF21 reduced blood glucose, blood lipid, and insulin levels, and improved glucose metabolism and insulin sensitivity ${ }^{36-41}$. Therefore, our data suggest that FGF21 affects glucose metabolism in LNCaP cells, for example, it may inhibit the utilization of glucose in LNCaP cells and thereby inhibits their proliferation. In addition, we found that the tumor volume in mice implanted with high glucose-treated LNCaP cells was greater than that in mice implanted with low glucosetreated cells. Indeed, growing evidence has shown that cells exposed to a hyperglycemic environment can affect the change in gene expression through epigenetic modifications, and this effect is sustained even if there is no hyperglycemia environment, which is called hyperglycemic memory $^{42,43}$. The epigenetic modification of genes is very important for PCa progress ${ }^{44,45}$. Therefore, we thus believe that the epigenetic modification of gene expression in the LNCaP cells may be the reason for their faster growth in the nude mice.

Autophagy is a cellular process that degrades intracellular organelles and proteins to maintain cellular homeostasis $^{46,47}$. Autophagy-related cell death is considered an important mechanism of cell death ${ }^{48,49}$. It was recently proved that autophagy can regulate glucose and lipid metabolism in tissues and cells ${ }^{50}$ and affects tumorigenesis $^{51}$. Indeed, the activation or inhibition of autophagy plays an essential role in tumor growth ${ }^{52,53}$. Here, we observed that FGF21 overexpression increased LC3-I conversion into LC3-II, and the expression of LAMP2 protein (a well-known marker of autophagosome formation $)^{54}$ reduced p62 levels and increased autophagosome formation in LNCaP cells, which suggests that FGF21 facilitates autophagy. Autophagy has also recently been found to be vital for inhibiting proliferation and promoting apoptosis in tumor cells ${ }^{5,56}$. Therefore, we speculate that FGF21 may inhibit the proliferation of PCa cells and trigger apoptosis through autophagy.

An increasing number of reports show that the PI3k-Akt signaling pathway plays a major role in tumor cell apoptosis and proliferation ${ }^{57}$. In addition, mTOR, a downstream mediator of the PI3K-Akt pathway, is related to the regulation of autophagy ${ }^{58}$. Other studies have also shown that the PI3K/Akt/mTOR signaling pathway exerts an antiapoptotic effect and contributes to autophagic regulation in cancer cells ${ }^{59}$. However, how autophagy is related to the PI3K-AKT-mTOR pathway is not clear. Activated mTORC1 downregulates autophagy by phosphorylating a complex of autophagy proteins (unc-51-like autophagy activating kinase 1 [ULK1]/ ULK2), which inhibits the downstream autophagy cascade $^{60}$. Furthermore, Akt can be activated via phosphorylation by mTORC2, providing positive feedback that contributes to the inhibition of autophagy ${ }^{61}$. In addition, active Akt can directly regulate transcription factors FOXOs (forkhead box O proteins), resulting in the inhibition of autophagy ${ }^{62}$.

The relationship between FGF21 and the PI3K-Akt-mTOR pathway has been reported, but the results are not consistent ${ }^{63-65}$. Minard et al. ${ }^{63}$ reported that FGF21 activated mTORC1 via MAPK rather than through the canonical PI3K-Akt pathway. Further, FGF21 activates PI3K-Akt signaling, and in turn, $\mathrm{mTOR}^{66}$. On the contrary, Gong et al. ${ }^{65}$ found that FGF21 inhibited mTORC1 activity induced by nutrients and hormones. Therefore, the relationship between FGF21 and the mTOR signal remains unclear. Herein, KEGG analysis showed that FGF21 is related to the PI3K-Akt-mTOR signaling pathway. Therefore, we speculate that FGF21 promotes autophagy in LNCaP cells by modulating PI3KAkt-mTOR signaling. Consistent with this hypothesis, we observed that p-FGF21 treatment downregulated the phosphorylation levels of Akt, mTOR, and their downstream target molecule, P70S6K, in LNCaP cells, indicating that PI3K-Akt-mTOR signaling was inhibited. However, mTOR agonist treatment blocked the effects of FGF21 on the conversion of LC3-I to LC3-II, and on p62, $\mathrm{Ki}-67$, and BCL-2 protein expression. These data reveal that the PI3K-Akt-mTOR-p70S6K pathway plays a pivotal role in FGF21-induced autophagy and in regulating $\mathrm{LNCaP}$ cell proliferation and apoptosis. Indeed, an imbalance in the PI3K-Akt-mTOR pathway is a common mechanism leading to the occurrence and development of tumors ${ }^{67}$. Accordingly, our results present a possible mechanism for the role of FGF21 in PCa by modulating the PI3K-Akt-mTOR pathway.

As a secretory protein, FGF21 is mainly expressed and secreted in the liver under physiological conditions, but it is also expressed and secreted in other tissues under pathological conditions ${ }^{33,68}$. FGF21 can play a role in various tissues and organs of the human body through the circulatory system. Therefore, we believe that increasing FGF21 expression and secretion in PCa tissue or increasing circulating FGF21 level will have an important impact on the proliferation and apoptosis of PCa cells, and may become a new target for PCa treatment.

The limitations of this study are: (1) we could not determine the direct biological effects of FGF21, as there 
are differences between in vitro experiments and the human internal environment; (2) the effect of endogenous FGF21 on PCa remains unclear and requires further study; (3) KEGG analysis of the signaling pathway was performed on mouse adipose tissue, which may be different from prostate tissue. Moreover, we did not measure the circulating FGF21 levels in patients with PCa. Therefore, further studies are needed to determine whether FGF21 is a biomarker in patients with PCa. Nonetheless, one paper cannot solve all of the scientific problems. Despite these limitations, ours is the first study to discover the role of FGF21 in the proliferation, invasion, and apoptosis of $\mathrm{PCa}$ cells, and to suggest that FGF21 may be a molecular target for PCa therapy.

In summary, FGF21 is downregulated in PCa tissues and cell lines and can inhibit PCa cell proliferation and facilitate their apoptosis. GO and KEGG analyses showed that FGF21 might be related to the PI3K-Akt-mTOR signaling pathway and autophagy. Molecular biology experiments confirmed that FGF21 promoted PCa cell apoptosis through autophagy mediated by PI3K-Akt-mTOR signaling (Fig. 6E). Therefore, our results indicate that FGF21 is a potential new target for PCa therapy.

\section{Acknowledgements}

We thank the Department of Pathology, Chongqing Medical University for their skilled technical assistance.

\section{Author details}

${ }^{1}$ The Key Laboratory of Laboratory Medical Diagnostics in the Ministry of Education and Department of Clinical Biochemistry, College of Laboratory Medicine, Chongqing Medical University, Chongqing, China. ${ }^{2}$ Department of Endocrinology, The Second Affiliated Hospital, Chongqing Medical University, Chongqing, China. ${ }^{3}$ Chongqing Prevention and Treatment Hospital for Occupational Diseases, Chongqing, China

\section{Author contributions}

H.D., W.H., L.Z., F.J., and X.M. collected experimental data and performed this study. G.Y. participated in data interpretation and manuscript preparation. L.L. is the person who takes full responsibility for the work as a whole, including the study design, access to data, and the decision to submit and publish the manuscript.

\section{Funding}

This work was supported by Grants from the National Natural Science Foundation of China (Nos. 81873658, 81601214, and 81700758).

\section{Data availability}

All data included in this study are available and include, where applicable, accession codes, other unique identifiers and associated web links for publicly available datasets, and any conditions for access of non-publicly available datasets

\section{Ethics}

All in vitro and in vivo experiments were approved in advance by the Chongqing Medical University Ethics Committee and Chongqing Medical University Animal Experimentation Ethics Committee.

\section{Conflict of interest}

The authors declare no competing interests.

\section{Publisher's note}

Springer Nature remains neutral with regard to jurisdictional claims in published maps and institutional affiliations.

Supplementary information The online version contains supplementary material available at https://doi.org/10.1038/s41419-021-03588-w.

Received: 29 October 2020 Revised: 27 February 2021 Accepted: 3 March 2021

Published online: 22 March 2021

\section{References}

1. Siegel, R. L., Miller, K. D. \& Jemal, A. Cancer statistics, CA. Cancer J. Clin. 68, 7-30 (2018).

2. Gu, X. Y. et al. Analysis on the trend of prostate cancer incidence and age change in cancer registration areas of China, 2000 to 2014. Chin. J. Prev. Med. 52, 586-592 (2018).

3. Grasso, C. S. et al. The mutational landscape of lethal castration-resistant prostate cancer. Nature 487, 239-243 (2012).

4. Ornitz, D. M. \& Itoh, N. Fibroblast growth factors. Genome Biol. 2, Reviews3005 (2001).

5. Beenken, A. \& Mohammadi, M. The FGF family: biology, pathophysiology and therapy. Nat. Rev. Drug. Discov. 8, 235-8253 (2009).

6. Potthoff, M. J., Kliewer, S. A. \& Mangelsdorf, D. J. Endocrine fibroblast growth factors 15/19 and 21: from feast to famine. Genes Discov. 26, 312-324 (2012).

7. Song, K. H., Li, T., Owsley, E., Strom, S. \& Chiang, J. Y. Bile acids activate fibroblast growth factor 19 signaling in human hepatocytes to inhibit cholesterol 7alpha-hydroxylase gene expression. Hepatology 49, 297-305 (2009).

8. $\mathrm{Xu}$, J. et al. Acute glucose-lowering and insulin-sensitizing action of FGF21 in insulin-resistant mouse models-association with liver and adipose tissue effects. Am. J. Physiol. Endocrinol. Metab. 297, E1105-E1114 (2009).

9. $\mathrm{Xu}$, J. et al. Fibroblast growth factor 21 reverses hepatic steatosis, increases energy expenditure, and improves insulin sensitivity in diet-induced obese mice. Diabetes 58, 250-259 (2009).

10. Bhatnagar, S., Damron, H. A. \& Hillgartner, F. B. Fibroblast growth factor-19, a novel factor that inhibits hepatic fatty acid synthesis. J. Biol. Chem. 284, 10023-10033 (2009)

11. Qian, J., Tikk, K, Weigl, K, Balavarca, Y. \& Brenner, H. Fibroblast growth factor 21 as a circulating biomarker at various stages of colorectal carcinogenesis. Br. J. Cancer 119, 1374-1382 (2018).

12. Kang, Y. E. et al. Association between circulating fibroblast growth factor 21 and aggressiveness in thyroid cancer. Cancers 11, 1154 (2019).

13. Singhal, G. et al. Deficiency of fibroblast growth factor 21 (FGF21) promotes hepatocellular carcinoma (HCC) in mice on a long term obesogenic diet. Mol. Metab. 13, 56-66 (2018)

14. Nagamatsu, H. et al. GF19 promotes progression of prostate cancer. Prostate 75, 1092-1101 (2015)

15. Feng, S., Wang, J., Zhang, Y., Creighton, C. J. \& Ittmann, M. FGF23 promotes prostatecancer progression. Oncotarget 6, 17291-17301 (2015).

16. Moch, H., Cubilla, A. L., Humphrey, P. A., Reuter, V. E. \& Ulbright, T. M. The 2016 WHO Classification of tumours of the urinary system and male genital organspart a: renal, penile, and testicular tumours. Eur. Urol. 70, 93-105 (2016).

17. Epstein, J. I. et al. The 2014 International Society of Urological Pathology (ISUP) Consensus Conference on Gleason Grading of Prostatic Carcinoma: definition of grading patterns and proposal for a new grading system. Am. J. Surg. Pathol. 40, 244-252 (2016).

18. Xie, Z. C. et al. Investigation of miR-136-5p key target genes and pathways in lung squamous cell cancer based on TCGA database and bioinformatics analysis. Pathol. Res. Pract. 214, 644-654 (2018).

19. $L i, K$. et al. The effects of fibroblast growth factor-21 knockdown and overexpression on its signaling pathway and glucose-lipid metabolism in vitro. Mol. Cell. Endocrinol. 348, 21-26 (2012).

20. Jia, Y. et al. Effective gene delivery of shBMP-9 using polyethyleneimine-based core-shell nanoparticles in an animal model of insulin resistance. Nanoscale 11, 2008-2016 (2019)

21. Zhang, C. et al. Osteoprotegerin promotes liver steatosis by targeting the ERKPPARgamma-CD36 pathway. Diabetes 68, 1902-1914 (2019).

22. Lai, Y. et al. DOCK5 regulates energy balance and hepatic insulin sensitivity by targeting mTORC1 signaling. EMBO Rep. 21, e49473 (2020). 
23. Zhou, M. et al. Effect of central JAZF1 on glucose production is regulated by the PI3K-Akt-AMPK pathway. FASEB J. 34, 7058-7074 (2020).

24. Rahn, S. et al. Diabetes as risk factor for pancreatic cancer: hyperglycemia promotes epithelial-mesenchymal-transition and stem cell properties in pancreatic ductal epithelial cells. Cancer Lett. 415, 129-150 (2018).

25. Zhou, Q. et al. MicroRNA-20a regulates cell proliferation, apoptosis and autophagy by targeting thrombospondin 2 in cervical cancer. Eur. J. Pharm. 844, 102-109 (2019).

26. Russell, R. C., Yuan, H. X. \& Guan, K. L. Autophagy regulation by nutrient signaling. Cell Res. 24, 42-57 (2014).

27. Yang, L. et al. Palmitic acid induces human osteoblast-like Saos-2 cell apoptosis via endoplasmic reticulum stress and autophagy. Cell Stress Chaperones 23 1283-1294 (2018)

28. Turner, N. \& Grose, R. Fibroblast growth factor signalling: from development to cancer. Nat. Rev. Cancer 10, 116-129 (2010).

29. Wesche, J., Haglund, K. \& Haugsten, E. M. Fibroblast growth factors and their receptors in cancer. Biochem. J 437, 199-213 (2011).

30. Acevedo, V. D. et al. Inducible FGFR-1 activation leads to irreversible prostate adenocarcinoma and an epithelial-to-mesenchymal transition. Cancer Cell. 12, 559-571 (2007).

31. Marek, L. et al. Fibroblast growth factor (FGF) and FGF receptor-mediated autocrine signaling in non-small-cell lung cancer cells. Mol. Pharm. 75 196-207 (2009).

32. Vazquez, A. et al. Cancer metabolism at a glance. J. Cell Sci. 129, 3367-3373 (2016).

33. Yang, C. et al. Activation of Liver FGF21 in hepatocarcinogenesis and during hepatic stress. BMC Gastroenterol. 13, 67 (2013).

34. Akyol, M. et al. The alterations of serum FGF-21 levels, metabolic and body composition in early breast cancer patients receiving adjuvant endocrine therapy. Cancer Biomark. 18, 441-449 (2017).

35. Knott, M. E. et al. Circulating fibroblast growth factor 21 (Fgf21) as diagnostic and prognostic biomarker in renal cancer. J. Mol. Biomark. Diagn. 1, (Suppl 2):015 (2016).

36. Kharitonenkov, A. et al. FGF-21 as a novel metabolic regulator. J. Clin. Invest. 115, 1627-1635 (2005).

37. Coskun, T. et al. Fibroblast growth factor 21 corrects obesity in mice. Endocrinology 149, 6018-6027 (2008).

38. Berglund, E. D. et al. Fibroblast growth factor 21 controls glycemia via regulation of hepatic glucose flux and insulin sensitivity. Endocrinology 150, 4084-4093 (2009).

39. Adams, A. C. et al. Fundamentals of FGF19 \& FGF21 action in vitro and in vivo. PLOS ONE 7, e38438 (2012).

40. Adams, A. C. et al. The breadth of FGF21's metabolic actions are governed by FGFR1 in adipose tissue. Mol. Metab. 2, 31-37 (2012).

41. Kharitonenkov, A. et al. The metabolic state of diabetic monkeys is regulated by fibroblast growth factor-21. Endocrinology 148, 774-781 (2007).

42. Vasconcelos-Dos-Santos, A. et al. Hyperglycemia exacerbates colon cancer malignancy through hexosamine biosynthetic pathway. Oncogenesis 6, e306 (2017).

43. Plass, C. et al. Mutations in regulators of the epigenome and their connections to global chromatin patterns in cancer. Nat. Rev. Genet. 14, 765-780 (2013).

44. Biernacka, K. M. et al. Hyperglycaemia-induced chemoresistance of prostate cancer cells due to IGFBP2. Endocr. Relat. Cancer 20, 741-751 (2013).

45. Lee, C., An, D. \& Park, J. Hyperglycemic memory in metabolism and cancer. Horm. Mol. Biol. Clin. Investig. 26, 77-85 (2016).

46. Bai, J. \& Liu, F. The CGAS-cGAMP-STING pathway: a molecular link between immunity and metabolism. Diabetes 68, 1099-1108 (2019).
47. Mizushima, N. Physiological functions of autophagy. Curr. Top. Microbiol. Immunol. 335, 71-84 (2009).

48. Yang, Z. J., Chee, C. E., Huang, S. \& Sinicrope, F. A. The role of autophagy in cancer: therapeutic implications. Mol. Cancer Ther. 10, 1533-1541 (2011).

49. Mizushima, N., Levine, B., Cuervo, A. M. \& Klionsky, D. J. Autophagy fights disease through cellular selfdigestion. Nature 451, 1069-1075 (2008).

50. Xiao, Y. et al. Activation of ERK1/2 ameliorates liver steatosis in leptin receptordeficient $(\mathrm{db} / \mathrm{db})$ mice via stimulating ATG7-dependent autophagy. Diabetes 65, 393-405 (2016).

51. Gao, S. et al. MiR-146b inhibits autophagy in prostate cancer by targeting the PTEN/Akt/mTOR signaling pathway. Aging 10, 2113-2121 (2018).

52. Chen, S. et al. MiR-144 inhibits proliferation and induces apoptosis and autophagy in lung cancer cells by targeting TIGAR. Cell Physiol. Biochem. 35 997-1007 (2015).

53. De Amicis, F. et al. A novel functional interplay between progesterone receptor- and PTEN, via AKT, modulates autophagy in breast cancer cells. J. Cell. Mol. Med. 18, 2252-2265 (2014).

54. Saftig, P., Schroder, B. \& Blanz, J. Lysosomal membrane proteins: life between acid and neutral conditions. Biochem. Soc. Trans. 38, 1420-1423 (2010).

55. Ma, J. F. et al. The ethyl acetate extract of gynura formosana kitam. leaves inhibited cervical cancer cell proliferation via induction of autophagy. Biomed. Res. Int. 24, 4780612 (2018).

56. Patra, S. et al. Dysregulation of histone deacetylases in carcinogenesis and tumor progression: a possible link to apoptosis and autophagy. Cell Mol. Life Sci. 76, 3263-3282 (2019).

57. Ishrat, T., Sayeed, I., Atif, F., Hua, F. \& Stein, D. G. Progesterone is neuroprotective against ischemic brain injury through its effects on the phosphoinositide 3kinase/protein kinase B signaling pathway. Neuroscience 210, 442-450 (2012).

58. Hu, Z., Yang, B., Mo, X. \& Xiao, H. Mechanism and regulation of autophagy and its role in neuronal diseases. Mol. Neurobiol. 52, 1190-1209 (2015).

59. Kumar, D., Shankar, S. \& Srivastava, R. K. Rottlerin induces autophagy and apoptosis in prostate cancer stem cells via PI3K/Akt/mTOR signaling pathway. Cancer Lett. 343, 179-189 (2014).

60. Janku, F., McConkey, D. J., Hong, D. S. \& Kurzrock, R. Autophagy as a target for anticancer therapy. Nat. Rev. Clin. Oncol. 8, 528-539 (2011).

61. Sun, S. Y. et al. Activation of Akt and elF4E survival pathways by rapamycinmediated mammalian target of rapamycin inhibition. Cancer Res. 65, 7052-7058 (2005)

62. Liu, L., Liao, J. Z., He, X. X. \& Li, P. Y. The role of autophagy in hepatocellular carcinoma: friend or foe. Oncotarget 8, 57707-57722 (2017).

63. Minard, A. Y. et al. mTORC1 is a major regulatory node in the FGF21 signaling network in adipocytes. Cell Rep. 17, 29-36 (2016).

64. Alonge, K. M., Meares, G. P. \& Hillgartner, F. B. Glucagon and insulin cooperatively stimulate fibroblast growth factor 21 gene transcription by increasing the expression of activating transcription factor 4. J. Biol. Chem. 292, 5239-5252 (2017).

65. Gong, Q. et al. Fibroblast growth factor 21 improves hepatic insulin sensitivity by inhibiting mammalian target of rapamycin complex 1 in mice. Hepatology 64, 425-438 (2016).

66. Yu, X. et al. FGF21 promotes non-small cell lung cancer progression by SIRT1/ PI3K/AKT signaling. Life Sci. 269, 118875 (2020).

67. Wong, K. K., Engelman, J. A. \& Cantley, L. C. Targeting the PI3K signaling pathway in cancer. Curr. Opin. Genet. Dev. 20, 87-90 (2010).

68. Singhal, G. et al. Fibroblast growth factor 21 (FGF21) protects against high fat diet induced inflammation and islet hyperplasia in pancreas. PLOS ONE $\mathbf{1 1}$ e0148252 (2016). 